\title{
Erratum to: Risk of cardiovascular events among patients with HIV treated with atazanavircontaining regimens: a retrospective cohort study
}

Lisa Rosenblatt ${ }^{1}$, Amanda M. Farr² ${ }^{2 *}$ Ella T. Nkhoma ${ }^{3}$, James K. Nelson ${ }^{4}$, Corey Ritchings ${ }^{5}$ and Stephen S. Johnston ${ }^{6}$

\section{Erratum}

n.b. The errors and associated corrections described in this document concerning the original manuscript were accountable to the production department handling this manuscript, and thus are no fault of the authors of this paper. Additionally, the online manuscript has now been updated with these corrections accordingly.

In the original publication of this article [1], Fig. 3 had [VALUE] $(0.72,2.23)$ where it should have said $1.27(0.72$, 2.23). This has now been updated in the original article.

\footnotetext{
* Correspondence: amanda.farr@truvenhealth.com

${ }^{2}$ Truven Health Analytics, 150 Cambridgepark Drive, Cambridge, MA 02140, USA

Full list of author information is available at the end of the article
} 


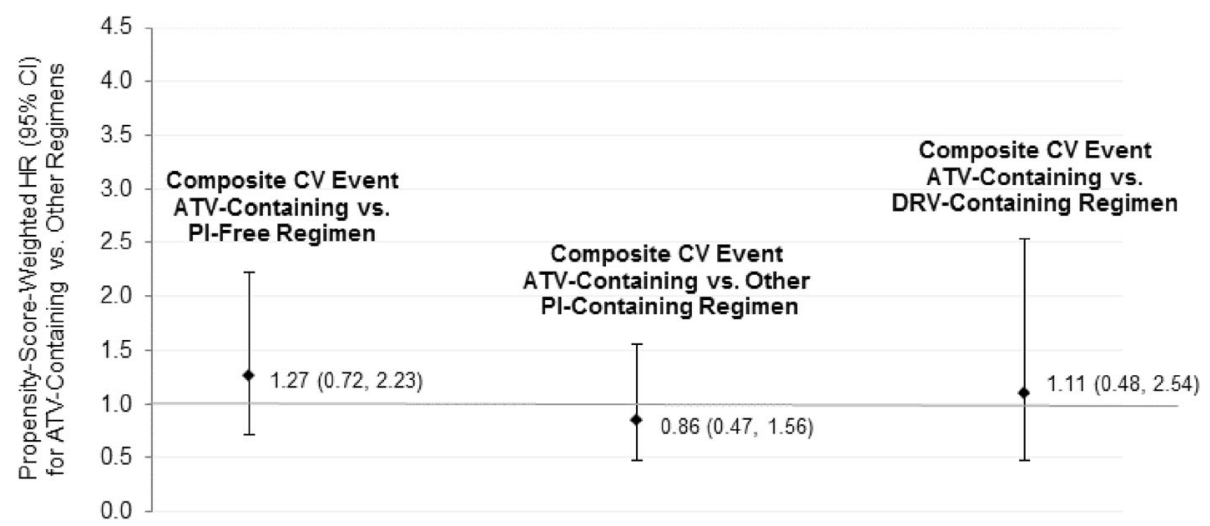

Fig. 3 Propensity-score-weighted hazard ratios for composite CV event during as-treated follow-up period: Secondary comparisons. Cl confidence interval, CV cardiovascular, DRV darunavir, HR hazard ratio, PI protease inhibitor

\section{Author details}

'Bristol-Myers Squibb, 777 Scudders Mill Road, Plainsboro, NJ 08536, USA.

${ }^{2}$ Truven Health Analytics, 150 Cambridgepark Drive, Cambridge, MA 02140,

USA. ${ }^{3}$ Bristol-Myers Squibb, 5 Research Parkway, Wallingford, CT 06492, USA

${ }^{4}$ Truven Health Analytics, 100 Phoenix Drive, Ann Arbor, MI 48108, USA.

${ }^{5}$ Bristol-Myers Squibb, PO Box 4500, Princeton, NJ 08540, USA. ${ }^{6}$ Truven Health

Analytics, 7700 Old Georgetown Road, Bethesda, MD 20814, USA.

Received: 27 September 2016 Accepted: 5 October 2016

Published online: 07 October 2016

\section{References}

1. Rosenblatt L, et al. Risk of cardiovascular events among patients with HIV treated with atazanavircontaining regimens: a retrospective cohort study. BMC Infect Dis. 2016;16:492. doi:10.1186/s12879-016-1827-1. 